|| ISSN(online): 2589-8698 || ISSN(print): 2589-868X ||

International Journal of Medical and Biomedical Studies

Available Online at www.ijmbs.info

NLM (National Library of Medicine ID: 101738825)

Index Copernicus Value 2020: 79.44

Original Research Article

Volume 6, Issue 02; February: 2022; Page No. $45-46$

\title{
PREVALENCE OF POSITIVITY OF CBNAAT IN EXTRA PULMONARY TUBERCULOSIS
}

\author{
Dr Rakesh Kumar Bhatnagar
}

MBBS, MD (TB \& Respiratory Diseases), Principal Chief Medical Officer, Chittorgarh (Rajasthan)

Article Info: Received 03 January 22; Accepted 16 February 2022

DOI: https://doi.org/10.32553/ijmbs.v6i2.2440

Corresponding author: Dr Rakesh Kumar Bhatnagar

Conflict of interest: No conflict of interest.

\section{Abstract}

Background: TB is the $9^{\text {th }}$ leading cause of death worldwide and the leading cause from a single infectious agent. It is estimated that 15 to $20 \%$ of all TB cases are extra pulmonary.

Methods: This study conducted on Patients of EPTB diagnosed on the basis of clinico radiological basis attending the OPD. Patients with signs and symptoms and radiological examination suggestive of extra-pulmonary tuberculosis were included in this study.

Results: In present study $16(64.00 \%)$ patients suggestive of TB by FNAC were CBNAAT positive and 9(36.00\%) were CBNAAT negative. 5 patients $(45.45 \%)$ were NOT suggestive of TB by FNAC were CBNAAT negative and 6(54.55\%) were CBNAAT positive. This difference however is not found to be statistically significant $(\mathrm{p}=0.869)$.

Conclusion: Present study revealed that more positivity rate by CBNAAT in comparision to smear staining in pleural fluid which indicate that it is a more sensitive technique as compared to conventional methods.

Keywords: CBNAAT, Extrapulmonary tuberculosis, FNAC

\section{Introduction}

India is the home of world's largest tuberculosis (TB) burden, accounting for around $21 \%$ of the TB incidence globally. Although pulmonary involvement is the most common presentation, it can potentially affect any organ or system of the body. Extra pulmonary tuberculosis is defined according to WHO classification criteria as an infection by $\mathrm{M}$. tuberculosis which affects tissues and organs outside the pulmonary parenchyma. ${ }^{1}$

In India, EPTB constitutes $10-15 \%$ of total TB cases which primarily involve the pleura, lymph nodes, gastrointestinal tract and other organs with a significant case mortalityrate (25 to $50 \%)^{2}$

More recently a number of studies were done to evaluate this assay using non-respiratory clinical samples from patients suspected of having EPTB., ${ }^{3,4}$ In 2014, WHO has recommended CBNAAT over the conventional tests (including conventional microscopy, culture or histopathology) for testing specific non-respiratory specimens (lymph nodes and other tissues) from patients suspected of having EPTB. ${ }^{5}$ However, this was a conditional recommendation due to very low-quality evidence available. More studies are therefore needed particularly in settings with high EPTB prevalence.

\section{Material and Methods}

STUDY DESIGN- This was a observational study.
Patients of extrapulmonary Tuberculosis diagnosed on the basis of clinico radiological basis attending the outpatient clinic of the department of pulmonary medicine of our hospital and The protocol was explained to the patient/care provider before enrolment and informed consent were taken from each patient.

Patients who attended department of pulmonary medicine with signs and symptoms and radiological examination suggestive of extra-pulmonary tuberculosis were included in this study. Their detailed clinical history, demographic profile, socioeconomic status and contact number was taken and recorded. Previous history of tuberculosis, past history of medical illness and h/o co-morbid illnesses were also recorded. A fresh digital chest radiograph was advised to study population with suspected pleural effusion, hydropneumothorax or pyo-pneumothorax. A new chest X-ray was also advised for patients who did not had their old chest radiograph.

\section{Inclusion Criteria}

1. All cases of lymphadenopathy diagnosed on the basis of clinico radiological and/or histopathological findings.

2. Patient who gave informed consent.

Exclusion Criteria

1. Cases of malignant $\&$ inflammatory lymphadenopathy

2. Patient who did not give informed consent 


\section{Observations}

Table 1: CBNAAT result relation to FNAC result in Lymph node sample

\begin{tabular}{|l|l|l|l|l|l|l|}
\hline \multirow{2}{*}{ FNAC result } & CBNAAT positive & \multicolumn{4}{l|}{ CBNAAT negative } & Total \\
\cline { 2 - 7 } & $\mathrm{N}$ & $\%$ & $\mathrm{~N}$ & $\%$ & $\mathrm{~N}$ & $\%$ \\
\hline Suggestive of TB & 16 & 64.00 & 9 & 36.00 & 25 & 100.00 \\
\hline Not suggestive of TB & 6 & 54.55 & 5 & 45.45 & 11 & 100.00 \\
\hline Total & 22 & 61.11 & 14 & 38.89 & 36 & 100.00 \\
\hline
\end{tabular}

Chi-square $=0.027$ with 1 degree of freedom; $p=0.869$ (NS)

In present study $16(64.00 \%)$ patients suggestive of TB by FNAC were CBNAAT positive and $9(36.00 \%)$ were CBNAAT negative. 5 patients $(45.45 \%)$ were NOT suggestive of TB by FNAC were CBNAAT negative and $6(54.55 \%)$ were CBNAAT positive. This difference however is not found to be statistically significant $(\mathrm{p}=0.869)$.

\section{Discussion}

Tuberculosis (TB) remains a key challenge in the face of global public health and inadequate diagnostic assays have hampered our chances to tackle this disease effectively. According to WHO there were 10 million new TB cases and 1.5 million TB death in 2018. Extra pulmonary tuberculosis accounts for $20 \%$ of total burden of tuberculosis globally it Is estimated that approximately 70 million people will die from tuberculosis within the next 20 years and it is because of inadequate measures for TB control As the number of bacilli are very less in extrapulmonary samples and because of difficulty in obtaining tissues from deep seated organs; diagnosis is delays In most cases. The CBNAAT marks an important development in the field of rapid molecular TB diagnostics. This assay was rapidly endorsed by WHO in December 2010 as a replacement for sputum smear microscopy, particularly in setting with high rates of HIVassociated TB and multidrug resistant TB developed for testing sputum samples. ${ }^{5}$ This multifunctional diagnostic platform is an automated, closed system that perform real time PCR and can be used by operator with minimal technical expertise, enabling diagnosis of $\mathrm{TB}$ and simultaneous assessment of rifampicin resistance to be completed within 2 hours. This test detects DNA specific for Mycobacterium tuberculosis by polymerase chain reaction. Extra-pulmonary TB is far more complex because of the diversity of clinical sample types, difficulties in obtaining adequate tissue for analysis and in the extraction of $\mathrm{M}$. tuberculosis DNA from the samples. As there are limited studies about the use and efficacy of the CBNAAT in extra- pulmonary tuberculosis there is a need for more and more research on this novel approach. This study explores the use of CBNAAT in different non respiratory samples.

In the study by Singh $\mathrm{KG}$ et $\mathrm{al}^{5}$, total number of patients in this study was 57 in which FNA for cytology and CB-NAAT was done. CBNAAT was positive from lymph node in 43 out of the 47 FNAC positive patients, in 10 patients in whom FNAC was negative, CB-NAAT did not diagnose TBLN in 9 of them.

\section{Conclusion}

Present study revealed that more positivity rate by CBNAAT in comparision to smear staining in pleural fluid which indicate that it is a more sensitive technique as compared to conventional methods.

\section{References}

1. Takhar RP. NAAT: A new ray of hope in the early diagnosis of EPTB. Emerg Med (Los Angel). 2016;6:328.

2. Lee JY. Diagnosis and treatment of extrapulmonary tuberculosis. Tuber Res Dis (Seoul). 2015 Apr;78(2):4755.

3. Bekedam HJ1, Boeree M, Kamenya A, Liomba G, Ngwira B, Subramanyam VR, Harries AD. Tuberculous lymphadenitis, a diagnostic problem in areas of high prevalence of HIV and tuberculosis. Trans R Soc Trop Med Hyg. 1997 MayJun;91(3):294-7.

4. Annam V, Karigoudar MH, Yelikar BR. Improved microscopical detection of acid-fast bacilli by the modified bleach method in lymphnode aspirates. Indian J PatholMicrobiol. 2009;52:349-52

5. Singh KG, Tandon S, Nagdeote S.T, Sharma K, Kumar A. Role of CB-NAAT in diagnosing Mycobacterial tuberculosis and rifampicin resistance in tubercular peripheral lymphadenopathy. Int J Med Res Rev 2017;5(03):242-246 doi:10.17511/ijmrr. 2017.i03.05. 